\title{
Numerical Modeling of Flow-Driven Piezoelectric Energy Harvesting Devices
}

\author{
S. Ravi and A. Zilian
}

\begin{abstract}
The present work proposes uniform and simultaneous computational analysis of smart, low power energy harvesting devices targeting flow-induced vibrations in order to enable reliable sensitivity, robustness and efficiency studies of the associated nonlinear system involving fluid, structure, piezo-ceramics and electric circuit. The article introduces a monolithic approach that provides simultaneous modeling and analysis of the coupled energy harvester, which involves surfacecoupled fluid-structure interaction, volume-coupled piezoelectric mechanics and a controlling energy harvesting circuit for applications in energy harvesting. A spacetime finite element approximation is used for the numerical solution of the governing equations of the flow-driven piezoelectric energy harvesting device. This method enables modeling of different types of structures (plate, shells) with varying cross sections and material compositions, and different types of simple and advanced harvesting circuits.
\end{abstract}

\section{Introduction}

Energy harvesting is the process of generating usable electrical energy acquired from various ambient energy sources such as solar, thermal, fluid and mechanical vibrations that surround a system. A steady increase in the growth of wireless and portable electronic devices has led to the development of sophisticated lowpower micro-electromechanical devices (MEMS) such as sensors and actuators. The portable nature of these devices necessitates their ability to carry their own power supply. The aim of energy harvesting is to scavenge energy from the environment to

Srivathsan Ravi

University of Luxembourg, e-mail: srivathsan.ravi@uni.lu

Andreas Zilian

University of Luxembourg, e-mail: andreas.zilian@uni.lu 
power these electronic devices. Such harvesting methods provide significant incentives to replace batteries as power source for providing electrical energy because of the limited lifespans of batteries, and persistent stagnation in the technological development of batteries over the years. Many applications related to wireless sensor networks and low power miniature sensors require them to be fully embedded in the structure and placed in remote locations. Conventional power sources like batteries are not an option for applications where the devices need to have their own power supply for an indefinite period of time and are only periodically maintained.

There are many methods to obtain useful electrical energy from the ambient vibration energy that usually goes untapped. Research interest towards developing energy-harvesting devices (EHDs) has grown rapidly over the past few years, and many methods have been proposed to make use of the ambient source to generate electrical power. Some of these methods include electrostatic generation, electromagnetic induction, dielectric elastomers, and piezoelectric materials. Energy harvesting from piezoelectric materials have gained significant attention, as is evident from the number of literature published every year in this field, due to their ability to convert mechanical energy from cyclic straining directly into useful electrical energy. For reviews on various forms of piezoelectric energy harvesting refer to [1, 2].

The present work proposes uniform and simultaneous computational analysis of smart, low power energy harvesting devices targeting flow-induced vibrations in order to enable reliable sensitivity, robustness and efficiency studies of the associated nonlinear system involving fluid, structure, piezo-ceramics and electric circuit. The article introduces a monolithic approach that provides simultaneous modeling and analysis of the coupled energy harvester, which involves surface-coupled fluidstructure interaction, volume-coupled piezoelectric mechanics and a controlling energy harvesting circuit for applications in energy harvesting. A Space-time finite element approximation is used for the numerical solution of the governing equations of the flow-driven piezoelectric energy harvesting device. This method enables modeling of different types of structures (plate, shells) with varying cross sections and material compositions, and different types of simple and advanced harvesting circuits. It should be noted that it is a common practice in modeling of piezoelectric energy harvesters to consider a simple resistor element as a harvesting circuit.

The outline of this article is as follows. The remainder of Sect. 1 introduces the concept of piezoelectric energy harvesters and provides a brief review of various types of modeling approaches to the problem of piezoelectric energy harvesting from base excitations. Sect. 2 gives a brief overview of modeling approaches for flow-driven piezoelectric energy harvesters. Sect. 3 starts with the modeling assumptions of the present study and proceeds to establish in the detail the strong form of the governing equations of the multi-physics problem. The coupling conditions are also explained in Sect. 3. In Sect. 4, the weak form of the governing equations are derived and Sect. 5 explains the nature of space-time interpolation with an illustration. The theoretical concepts established are then applied to the problem of piezoelectric energy harvesting from a piezoelectric bimorph subjected to base excitations and presented as a case study in Sect. 6 . 


\subsection{Harvesting mechanical vibrations}

The prevalence of mechanical vibrations has attracted significant research interest in vibration-based energy harvesting methods. Power generation from ambient mechanical vibrations usually constitutes the conversion of ambient mechanical vibration into useful electrical energy with the help of an EHD, to power other devices with low power requirements. Piezoelectric transduction offers many advantages over other power generating methods due to it's low form factor, high energy density, ease of integration into other systems, and its unique ability to convert cyclic straining of the material into electrical energy.

Piezoelectric materials exhibit accumulation of electric charges in response to mechanical strains which is known as direct piezoelectric effect. The piezoelectric effect is a reversible process, where the materials exhibit change in their shape on application of an electric field known as inverse piezoelectric effect. Prototypical piezoelectric EHDs are cantilevers with a seismic mass and are attached to another substrate layer. They can be employed in various modes based on the electric field orientation and the polarization direction. Utilization of a proper coupling mode is one of the ways to increase the amount of energy harvested from the piezoelectric material. Two coupling mode exist viz., the -31 mode and the -33 mode respectively. The former is characterized by the straining of the material in the direction perpendicular to the poling direction and the latter by the straining in the same direction as the poling direction. The cantilever with a seismic mass configuration facilitates a lower resonant frequency in the first bending mode, making it easy to match the resonant frequency of the structure to the ambient vibrations to obtain maximum power output. Such systems are capable of producing power output ranging from a few $\mu W$ to a few $m W$.

The performance of these piezoelectric devices depends on various factors like the type of piezoelectric material used, size of the harvesting device, mass distribution, shape of the structure, and vibration modes to name a few. The impact of different geometries on the power density of vibration energy harvesters was studied in [3]. Coupling coefficients, strain distribution, and vibration frequency were perceived as the three limiting factors in the field of piezoelectric power scavenging, and alternative geometries were proposed to address each of these limiting factors. The real world application space was deemed too limited for testing the design considerations to improve coupling co-efficients. The strain distribution in the geometry is improved by varying the width of a beam type structure for the full utilization of straining along the length. Experimentally a 30\% increase in power was observed for trapezoidal beam compared to cantilever beams. An experimental comparison of several types of active composite actuators for power generation was carried out in [4]. The study compares a type of macro-fiber composite called MFCs made of piezoelectric fiber composites (PFCs) and interdigitated electrodes to two other actuators called Quick Pack consisting of a monolithic piezo-layer with standard electrodes and another actuator called Quick Pack IDE with interdigitated electrodes. They were all attached to the same beams and excited at their first twelve natural frequencies. The results showed that the conventional Quick Pack with stan- 
dard electrodes was able to harvest significant energy generating $137 \mu \mathrm{W}$ at $64 \mathrm{~Hz}$ while the Quick Pack IDE and MFC produced $29 \mu \mathrm{W}$ and $12 \mu W$ respectively. It was concluded from the study that although the MFCs' fibrous structure itself was not detrimental to the harvesting capacity, it is the low capacitance due the interdigitated electrodes that deteriorates the power output. Hence the MFCs were found impractical to real-world applications even though they had higher coupling coefficients. It is evident that all design considerations are towards the maximization of the power output from the harvester as the scope of application widens with increase in power generation.

\subsection{Models of piezoelectric energy harvesting devices}

Over the years many mathematical models have been proposed for the modeling of piezoelectric energy harvesters ranging from simple SDOF (single degree of freedom) models with closed form solutions for the voltage output and vibration characteristics to more sophisticated analytical and numerical methods to address various aspects of modeling. Many of the early works employed simple SDOF models to predict the voltage response of piezoelectric energy harvesting devices driven by harmonic base excitations. Piezoelectric energy harvesters are usually attached to an external circuit that transforms the harvested energy into usable form. In [5], an equivalent circuit model was proposed to account for the harvesting circuit along with the modeling of the energy harvester. The method discussed the representation of the energy harvester electrically, and then combined with the electrical representation of the harvesting circuit and modeled together in SPICE simulator. This method facilitated the representation of non-linear circuit components but suffered the drawback of simplification necessary for the harvester to be represented electrically. This prevented accounting for any change in harvester's properties during operating conditions. In another model developed in[6], a coupled FEM-circuit method was presented to account for the modeling of electrical circuits where the energy harvester was modeled using finite elements and the coupled to the electrical part modeled using a SPICE simulator. This method had comparable advantages to the model in [5] but is computationally expensive and does not provide a realistic representation of the strongly-coupled physics.

One of the frequently addressed issues in the modeling of piezoelectric energy harvesters is capturing the effect of an attached harvesting circuit. This investigation has led to piezoelectric materials being used in passive shunt damping applications as well. Earlier studies modeled this impact as a viscous damping on the harvester which was a reasonable approximation only in the case of electromagnetic generators as pointed out in [7]. The physics of the piezoelectric system is much more complex, and the impact of a harvesting circuit seems too complex to be modeled as viscous damping. Since it is common understanding that maximum power is harvested at resonance, incorrect modeling of damping will lead to inaccurate result in predicting the frequency of the system. It was shown that the load-resistance depen- 
dent variation of the resonance frequency and amplification of the motion at opencircuit frequency are indicators for the need for better representation of the effect of harvesting circuits. In one of the most important works in the field, [7, 8] presents a mathematical model with distributed parameter solution based on Euler-Bernoulli assumptions, and comparisons with several SDOF models are made to point out the inaccuracies in popular SDOF models. Several flaws in SDOF models, ranging from the neglect of base rotary motion up to the simplified modeling of damping induced by piezoelectric coupling, are addressed in this work and correction factors are introduced, where necessary, to the SDOF models. In their work, the harmonic base excitation case is considered a particular solution of the general base excitation which includes superimposed rotary motion of the base as well. The study points out that the inertia due to rigid body motion was neglected in most of the SDOF models and hence a correction factor to this effect is proposed. The relative motion transmissibility function derived from the ratio of tip deflection to base deflection is used to form a non-dimensional basis for comparison of the model to SDOF models. It is shown that the error percentage as a function of dimensionless frequency was as high as $35 \%$ in SDOF model.

Only few piezoelectric energy harvesting applications, where the geometry of the energy harvesting device is simple, lend themselves to analytical solutions [9]. Most of the piezoelectric energy harvesting applications are complex, and numerical methods are needed to obtain the electromechanical response of such systems. A pioneering work on the finite element modeling of piezoelectric materials was presented by [10], where mechanical displacements and electrical potential were used as unknowns and both direct and inverse piezoelectric effect were included in the formulation. Since then numerous piezoelectric finite elements have been developed including beam, plate, shell and solid elements. Readers are encouraged to refer to [11] for a detailed review of different finite element models used to model vibration based piezoelectric energy harvesters. Many of the reviewed finite elements use displacements and electric potential as unknowns as suggested by [10] with a linear approximation of the electric potential through the thickness of the element. However it was shown in [12] that the electric potential has a second order component in bending.

Mixed and hybrid finite element formulations are presented in [13, 14, 15, 16]. These formulations contain additional unknown fields besides mechanical displacements and electrical potential which reduces locking phenomena and makes the elements less susceptible to mesh distortion. The most general formulation is presented by [13] and contains six independent unknown fields which are displacements, strains and stresses for the mechanical part and electric potential, electric field and dielectric displacements for the electric part. Further mixed formulations with three and four unknown fields are derived from the six field formulation. A six field formulation is used by $[15,16]$ with additional enhancements for strain and electric field which further reduce locking. 


\section{Flow driven piezoelectric energy harvesters}

A glance at recent surveys $[2,1]$ on piezoelectric energy harvesting indicates that much of the research work in this field is focused on harvesting energy from vibrations due to base excitations or the excitations of the structure to which the harvester is attached. This focus may be attributed to various sources of ambient energy in an urban environment, but alternative sources have to be identified in case of EHD devices placed in remote locations. One potential energy source in such locations is the kinetic energy of fluids, i.e wind or water, which cater to those requirements. In order to extract energy from fluid flows, the kinetic energy of the fluid must first be transformed into straining energy of the harvester, which is then converted into electrical energy and utilized with appropriate harvesting circuits. A key idea in fluid-driven piezoelectric energy harvesting is to utilize the flow energy through controlled aero- or hydro-elasticity phenomena. Traditionally, the idea is to avoid dangerous fluid-structure interactions. In flow-driven piezoelectric energy harvesting, potentially harmful fluctuations are harnessed to provide power supply to small-scale energy harvesting devices. However, the research on piezoelectric energy harvesters placed directly in the fluid flow is fairly limited. There are different mechanisms to convert the flow-energy into cyclic straining of the energy harvester.

One of the ways of harvesting energy from fluid flow is instability induced excitation caused by fluid-intrinsic physical properties. The self-exciting flow instability produces oscillating forces even if the structure is stationary (e.g. Kármán vortex street). A further amplification of the exciting force is possible for fluid-structure feedback. The concept of energy harvesting eel, where the cyclic straining of the harvester was achieved by water flow utilizing Kármán vortex sheets was introduced in [17]. This is one of the first works to study energy harvesting from fluid flow. The fluctuations of "eel" shaped polymer beams placed in the wake of a bluff body was investigated in this research. Tests were conducted on different membranes ranging from $0.1 \mathrm{~mm}$ to $0.7 \mathrm{~mm}$ placed in water channel running at speeds ranging between $0.05 \mathrm{~ms}^{-1}$ to $0.8 \mathrm{~ms}^{-1}$. Two different widths of the bluff bodies viz., $5.08 \mathrm{~cm}$ and $3.81 \mathrm{~cm}$ were used to create the vortex sheets. It was shown that the membranes exhibited lock-in behavior to he shedding of the bluff body when they oscillate with the same frequency as the undisturbed wake behind the body. The relationship indicating conditions for locking were derived from Euler-Bernoulli beam theory. It was suggested that the eels should have small stiffness so as not to dampen the oscillations. Though the literature provides PIV(particle image velocimetry) images to support their predictions, it fails to provide any insights into the electrical output and the type of coupling existing between the multi-physics domains. A similar study on energy harvesting eels placed in the wake of a bluff body was carried out in [18].

In addition to vibrations produced by Kármán vortex sheets, movement-induced excitation - caused by fluctuating flow forces resulting from movements of the vibrating structural part- also provides a way to utilize flow energy for EHDs. Small deviations from the equilibrium position of the structure induce a redistribution of impacting fluid forces, which further increases initial disturbances. This gives rise to ongoing transfer of flow energy to the structural oscillator and is called dynamic 
instability. Flutter response of a piezoelectrically damped cantilever pipe utilizing such flow instabilities was studied by [6]. Energy harvesting from fluid flows with attached electrical circuit was studied with a more comprehensive model by [19]. The study considered the case of harvesting energy in the wake of a circular cylinder at high Reynold's number. This study involved a combination of experimental and analytical model. This model also considered the three-way interactions of the fluid-flow, structure and the harvesting circuit at the same time in contrast to the most previous studies with loosely-coupled approach for emulating the real-life scenario. SDOF analytical model was chosen to represent the piezoelectric structure, and the coupling between the circuit and the harvester was considered only under the open-circuit condition which makes the model ineligible in predicting energy output for a finite circuit resistance.

\section{Model of a flow-driven piezoelectric EHD}

This section introduces the strong form of the equations that govern the fluid flow, the mechanical state and the electrical state of the flow-driven piezoelectric energy harvester. The coupled system consists of a piezoelectric structure placed in fluid flow, potentially in the wake of an arbitrarily shaped body, and connected to an electric circuit as shown in Fig. 1. The solid consists of a substrate structure sandwiched between piezoelectric patches. The individual piezoelectric patches are assumed to be covered with continuous electrodes with one voltage output per patch. The electrodes are connected to a harvesting circuit. The harvesting circuit is assumed to consist of a simple resistance across the electrodes covering the patches, which, as mentioned earlier, is a common practice in modeling piezoelectric energy harvesting devices.

The choice of the solution strategy to a physical problem usually drives the modeling assumptions. The article proposes a monolithic solution strategy to the coupled problem of flow-driven energy harvesting which is a strongly-coupled modeling approach. To this extent, a native coupling between the fluid and the structural domain is achieved by $3 D$ modeling of the piezoelectric thin structure formulated in terms of the structural velocity. This modeling approach also enables a straightforward application of constitutive models to piezoelectric coupling. In the present study, the flow is modeled as incompressible and viscous. Turbulence effects are not considered. Both the substrate structure and the piezoelectric material obey linear material laws.

The fluid flow is modeled with the incompressible Navier-Stokes equations, consisting of momentum and mass conservation equations, and is described using an Eulerian framework in the current configuration where the space-time domain $Q=\Omega \times I$ within the time interval $I=\left(t_{\mathrm{a}}, t_{\mathrm{b}}\right)$. The equations of the fluid domain are time-dependent and accommodates moving boundaries resulting from structural deformations. The behavior of the piezoelectric structure, and the circuit, within the time interval $I=\left(t_{\mathrm{a}}, t_{\mathrm{b}}\right)$ and occupying the space-time domain $Q_{0}=\Omega_{0} \times I$, is 
described using a Lagrangian description in reference configuration. The subscript "0" refers to the reference configuration.

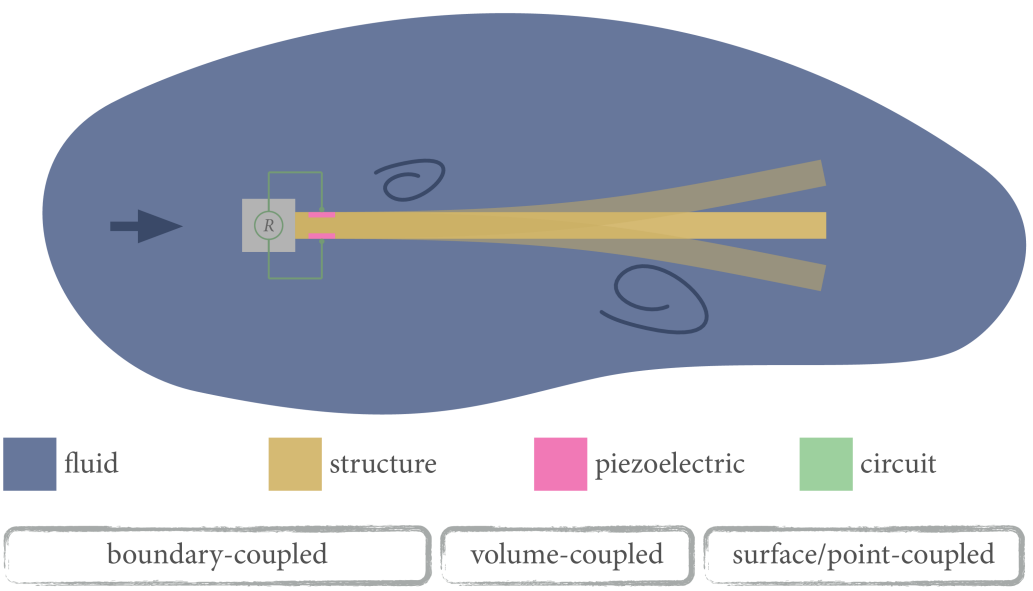

Fig. 1 An illustration of the multi-physics flow-driven piezoelectric EHD based on a cantilever setup.

\subsection{Fluid}

The incompressible Navier-Stokes equations describing the fluid flow are

$$
\rho(\dot{\mathbf{v}}+\mathbf{v} \cdot \nabla \mathbf{v})-\nabla \cdot \mathrm{T}-\mathbf{f}=\mathbf{0} \quad \text { in } \quad Q
$$

and

$$
\nabla \cdot \mathbf{v}=0 \quad \text { in } \quad Q
$$

where $\mathbf{v}$ is the velocity of the fluid, $\mathbf{f}$ is the external body force, and $\rho$ is the density of the fluid. The Cauchy stress tensor $\mathrm{T}$ is given by

$$
\mathrm{T}+p \mathrm{I}-2 \mu \mathrm{D}(\mathbf{v})=0 \quad \text { in } \quad Q
$$


where $\mu$ is the kinematic viscosity, $p$ is the hydrostatic pressure. The Cauchy stress tensor depends linearly on the strain rate tensor $\mathrm{D}$ given by

$$
\mathrm{D}(\mathbf{v})-\frac{1}{2}\left(\nabla \mathbf{v}+(\nabla \mathbf{v})^{\top}\right)=0 \quad \text { in } \quad Q
$$

with the boundary conditions being,

$$
\begin{array}{lll}
\mathbf{v}-\overline{\mathbf{v}}=0 & \text { on } \quad P^{\mathrm{v}} \quad \text { and } \\
\mathbf{t}-\overline{\mathbf{t}}=0 & \text { on } \quad P^{\mathrm{t}}
\end{array}
$$

where $P^{\mathrm{v}}$ in (5a) is the boundary on which velocity $\overline{\mathbf{v}}$ is imposed as a Dirichlet boundary condition, and $P^{\mathrm{t}}$ in (5b) is the boundary on which traction $\overline{\mathbf{t}}$ is imposed as a Neumann boundary conditions. The initial condition specifies a divergence free velocity field at time $t=0$

$$
\mathbf{v}(t=0)=\mathbf{v}_{\mathrm{i}} \quad \text { with } \quad \nabla \cdot \mathbf{v}_{\mathbf{i}}=0 \quad \text { on } \quad \Omega .
$$

\subsection{Piezoelectric structure}

The elastodynamic behavior of the piezoelectric structure is modeled based on the assumptions that the deformations are large and the material behavior is linear. The governing equations of the mechanical part of the electro-mechanically coupled piezoelectric structure are as follows

$$
\begin{gathered}
\rho_{0} \dot{\mathbf{v}}-\nabla_{0} \cdot(\mathrm{FS})-\mathbf{f}_{0}=\mathbf{0} \quad \text { in } Q_{0}, \\
\dot{\mathrm{E}}-\frac{1}{2}\left(\nabla_{0} \mathbf{v}+\left(\nabla_{0} \mathbf{v}\right)^{\top}+\left(\nabla_{0} \mathbf{u}\right)^{\top} \nabla_{0} \mathbf{v}+\left(\nabla_{0} \mathbf{v}\right)^{\top} \nabla_{0} \mathbf{u}\right)=0 \text { in } Q_{0}, \\
\dot{\mathrm{E}}-\left[s^{\tilde{\mathrm{D}}}\right] \dot{\mathrm{S}}-[g]^{\top} \dot{\tilde{\mathrm{D}}}_{0}=0 \quad \text { in } Q_{0},
\end{gathered}
$$

where (7) is the momentum balance equation, (8) gives the non-linear kinematic relation, and (9) depicts the coupled constitutive relation in rate form for the direct piezoelectric effect. $S$ is the second Piola-Kirchoff tensor, $\dot{E}$ is the strain rate tensor, $\left[s^{\tilde{D}}\right]$ is the compliance matrix measured at constant electric displacement, $[g]$ is the piezoelectric coefficient, and $\tilde{D}$ is the dielectric displacement of the piezoelectric structure.

Velocity $\overline{\mathbf{v}}$ is imposed as a Dirichlet boundary condition on $P_{0}^{\mathrm{v}}$ as

$$
\mathbf{v}-\overline{\mathbf{v}}=0 \quad \text { on } \quad P_{0}^{\mathrm{v}}
$$


whereas traction $\overline{\mathbf{t}}$ is imposed as a Neumann boundary condition on $P_{0}^{\mathrm{t}}$

$$
\mathbf{t}-\overline{\mathbf{t}}=0 \quad \text { on } \quad P_{0}^{t}
$$

The electromechanical behavior of the piezoelectric structure is described by Gauss' law which relates the distribution of electric charge to the electric field. A quasi-electrostatic approach is deemed adequate because the phase velocities of the acoustic waves are orders of magnitude less than the velocities of electromagnetic waves. The Gauss' law is given by

$$
\nabla_{0} \cdot \tilde{\mathrm{D}}_{0}=0 \quad \text { in } Q_{0} .
$$

The electrical field rate $\dot{\tilde{E}}$, is related to the electrical potential rate $\psi$, by the relation

$$
\dot{\tilde{\mathrm{E}}}_{0}+\nabla_{0} \psi=\mathbf{0} \text { in } Q_{0} .
$$

and the inverse piezoelectric constitutive equation in rate form is given by

$$
\dot{\tilde{\mathrm{E}}}+[g] \dot{\mathrm{S}}-\left[\varepsilon^{\mathrm{S}}\right]^{-1} \dot{\tilde{\mathrm{D}}}_{0}=\mathbf{0} \quad \text { in } \quad Q_{0}
$$

where the permittivity matrix, $\left[\varepsilon^{S}\right]^{-1}$, is measured at constant stress.

It is common in actuation and sensing applications of piezoelectric materials to impose electric potential and charge as Dirichlet and Neumann boundary conditions respectively. In the case of energy harvesting applications, however, both the electric potential and the electric charge are considered as unknowns. Most piezoelectric materials are manufactured with electrodes completely covering their top and bottom surfaces. Thus, a single potential output and charge output can be defined for individual piezoelectric patches. To this effect, appropriate Dirichlet and Neumann boundaries can be defined and a single potential and charge output can be assigned to them.

Electric potential rate $\bar{\psi}$ is given as a Dirichlet boundary condition on $P_{0}^{\psi}$ as

$$
\psi-\bar{\psi}=0 \quad \text { on } \quad P_{0}^{\psi}
$$

and electric charge $\bar{q}$ is given as a Neumann boundary condition on $P_{0}^{q}$ as

$$
q-\bar{q}=0 \quad \text { on } \quad P_{0}^{\mathrm{q}} .
$$

The electric potential rate $\bar{\psi}$, representing one of the two electrical variables defining individual piezoelectric patches, is further expressed as a single electrical potential output $\Phi_{\mathrm{p}}(t)$ of each piezoelectric patch.

It is pertinent to mention at this point, that the electrical field variables can be considered as analogous to the mechanical field variables. The electrical field variables charge and potential rate can be included in our intellection of generalized force and generalized structural velocity respectively. In the case of mixed hybrid 
model followed in this article, the analogy between electrical and mechanical field variables can be further extended to include electric field and electric displacement in our intellection of mechanical strain and mechanical stress respectively. This intellection provides for a clear comprehension of these quantities in a finite element framework.

\subsection{Circuit}

As mentioned earlier, individual piezoelectric patches are assumed to be covered with continuous electrodes. Free charges are localized on the electrode surface, and each electrode surface gives rise to a single voltage output. A harvesting circuit is attached to the electrodes, and the governing equations of the circuit are

$$
I-\dot{Q}=0 \quad \text { in } \quad I
$$

and

$$
\Delta \Phi-R \cdot I=0 \quad \text { in } \quad I
$$

where (17) is the charge conservation law. I is the current flowing through the circuit, and $Q$ is the electrical charge flowing though the circuit. Eq. (18) is the Ohm's law relating potential difference, $\Delta \Phi$ and the current flowing through the circuit. $R$ is the resistor element. $\Delta \Phi$ is the potential difference existing across the piezoelectric patches covering the substructure, and its value varies depending on the connection (series or parallel) between the patches.

\subsection{Coupling conditions}

Interface conditions determine how the different domains of the multi-physics system are coupled with each other, and depending on the interface conditions the modeling of the coupled domains can be either loosely coupled or strongly coupled. Since the research aims to have a strongly-coupled model of the flow-driven piezoelectric energy harvester, suitable interface conditions must be provided to represent the coupling between the fluid domain and the piezoelectric structural domain, and also the coupling between the electrical circuit and the harvester.

\subsubsection{Fluid-structure interface}

To complete the governing equations for the fluid-structure coupling consisting of a moving fluid-domain and vibrating elastic piezoelectric structure, coupling conditions have to be imposed on the interface $P^{\mathrm{C}}=P_{0}^{\mathrm{S}} \cap P^{\mathrm{F}}$ where the superscripts $S$ and 
$F$ denotes solid interface and fluid interface respectively. Geometrical continuity (or mass conservation) at the interface is achieved with the condition

$$
\mathbf{v}^{\mathrm{F}}-\mathbf{v}^{\mathrm{S}}=\mathbf{0} \quad \text { on } \quad P_{0}^{\mathrm{C}} .
$$

This leads to the momentum conservation enforced on the interface using the condition given by

$$
\mathbf{t}_{0}+\frac{d \Gamma_{\mathrm{t}}}{d \Gamma_{0}} \mathbf{t}^{\mathrm{F}}=\mathbf{0} \quad \text { on } \quad P_{0}^{\mathrm{C}}
$$

The above relation demands equal tractions along the deforming fluid-structure interface [20].

\subsubsection{Circuit-structure interface}

It is frequently assumed in many literature that the vibration characteristics of the energy harvesting device is independent of the electric circuit. However, as described in length in the previous sections, this assumption can lead to incorrect prediction of the harvester output. Piezoelectric sensors not connected to any circuit are usually modeled as current source in parallel with the capacitance of the piezoelectric material or a voltage source in series with the piezoelectric capacitance where, for the calculation of current source, the electric field $\tilde{E}$ is assumed as zero for short-circuit conditions, and the dielectric displacement $\tilde{D}$ is assumed zero for open-circuit calculations. But this condition is no more true in the case of an electric circuit attached directly to the piezoelectric structure. The circuit imposes a relation between the current flowing through the circuit and the voltage developed in the harvester due to the vibrations. This is given by the relation

$$
\Phi_{\mathrm{p}}(t)=\Phi_{\mathrm{R}}(t)
$$

where the voltage generated by the harvester $\Phi_{\mathrm{p}}(t)$ is assumed, a priori, equal to the voltage across the resistor element $\Phi_{\mathrm{R}}(t)$. The relation between this potential, and the current flowing through the resistor is given by equation (18). This relation is indicative of the strong-coupling between the circuitry and the structural domain, and provides a way to understand how an external circuit might impact the power generation capability of a harvesting device.

\section{Weak form of the governing equations}

In contrast to traditional finite element methods for elastodynamics where the solution is discretized in space, and solved in time domain using finite difference methods for ODE's leading a to semi-discrete formulation, the space-time finite element 
method facilitates consistent discretization of both the space and the time domain leading to a uniform discretization of the governing equations in their weighted residual form. The underlying concept of the space-time finite element method is to include the temporal axis where a space-time domain $Q$ is divided into $N$ time slabs as $Q_{\mathrm{n}}=\Omega_{\mathrm{n}} \times\left[t_{\mathrm{n}}, t_{\mathrm{n}+1}\right]$ as shown in Fig.2.
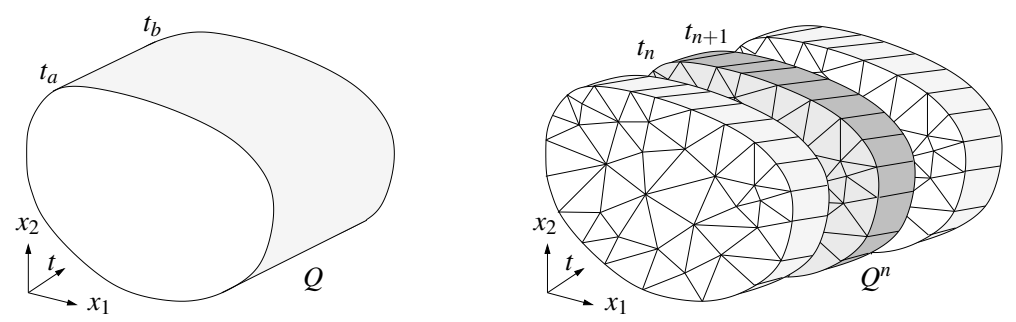

Fig. 2 Discretization of a domain with space-time finite elements using time slabs $Q_{\mathrm{n}}$, see [21].

The time integration is performed using a time-discontinuous Galerkin method. The information flow, as in conventional finite difference methods, is in the direction of positive time, and the discontinuous Galerkin method (DG method) leads to a system in which the solution to a time-slab $Q_{\mathrm{n}}=\Omega_{\mathrm{n}} \times\left[t_{\mathrm{n}}, t_{\mathrm{n}+1}\right]$ depends on the solution of the previous time at slab $t_{\mathrm{n}}^{-}$[22]. Hence the discontinuous approximation of the unknown fields in time leads to additional jump terms in the weak form which are derived in this section. The time integration scheme is implicit, A-Stable and third order accurate for linear interpolation in time [22]. For more detailed information on space-time finite elements readers are referred to [23, 22, 24].

\subsection{Fluid}

The Galerkin weighted residual form of the governing equations from (1)-(5b) describing the fluid flow are given as follows

$$
\begin{aligned}
& \int_{\mathrm{Q}_{\mathrm{n}}} \delta \mathbf{v} \cdot \rho(\dot{\mathbf{v}}+\mathbf{v} \cdot \nabla \mathbf{v}) \mathrm{d} Q+2 \mu \int_{\mathrm{Q}_{\mathrm{n}}} \mathrm{D}(\delta \mathbf{v}): \mathrm{D}(\mathbf{v}) \mathrm{d} Q \\
& -\int_{\mathrm{Q}_{\mathrm{n}}}(\nabla \cdot \delta \mathbf{v}) p \mathrm{~d} Q-\int_{\mathrm{Q}_{\mathrm{n}}} \delta \mathbf{v} \cdot \mathbf{f} \mathrm{d} Q \\
& +\int_{\mathrm{Q}_{\mathrm{n}}} \delta p(\nabla \cdot \mathbf{v}) \mathrm{d} Q \\
& +\int_{\Omega_{\mathrm{n}}} \delta \mathbf{v}\left(t_{\mathrm{n}}^{+}\right) \cdot \rho\left(\mathbf{v}\left(t_{\mathrm{n}}^{+}\right)-\mathbf{v}\left(t_{\mathrm{n}}^{-}\right)\right) \mathrm{d} \Omega_{\mathrm{t}} \\
& -\int_{\mathrm{P}_{\mathrm{n}}^{\mathrm{t}}} \delta \mathbf{v} \cdot \overline{\mathbf{t}} \mathrm{d} P=0 \quad \forall \delta \mathbf{v}, \delta p
\end{aligned}
$$


where (22a) represents the weighted form of the momentum conservation (1). Equation (3) describing the fluid constitutive equation, and equation (4) describing the fluid kinematics are satisfied exactly in equation (22a). Equation (22c) is the integral form of the mass conservation equation (2), weighted with the fluid pressure. The Neumann boundary condition (5b) is considered in a weak sense in equation (22e), while the Dirichlet condition is applied exactly a priori. Equation (22d) contains the jump terms required due to the time differentiation of the velocity using discontinuous Galerkin method, ensuring the transfer of kinetic energy from the end of previous time slab $t_{\mathrm{n}}^{-}$to the beginning of the current time slab at $t_{\mathrm{n}}^{+}$at time $t_{\mathrm{n}}$.

\subsection{Structure}

Unlike traditional displacement finite elements, velocity based mixed-hybrid finite elements for the structure allows for the native coupling at the fluid structure interface without having to resort to enforcement of continuity using Lagrange multipliers. This coupling strategy is followed in the model presented by this article, and hence the structural discretization is done using velocity based finite element method. The weighted residual form of the governing equations from (7)-(11) of the elastodynamics problem, but without piezoelectric coupling terms, and depicting the behavior of the elastic structure is given as,

$$
\begin{aligned}
& \int_{\mathrm{Q}_{0, \mathrm{n}}} \delta \mathbf{v} \rho_{0} \dot{\mathbf{v}} \mathrm{d} Q_{0}+\int_{\mathrm{Q}_{0, \mathrm{n}}} \dot{\mathrm{E}}(\delta \mathbf{v}, \mathbf{u}): \mathrm{Sd} Q_{0} \\
& -\int_{\mathrm{Q}_{0, \mathrm{n}}} \delta \mathbf{v} \cdot \mathbf{f}_{0} \mathrm{~d} Q_{0} \\
& +\int_{\mathrm{Q}_{0, \mathrm{n}}} \delta \mathrm{S}:([s] \dot{\mathrm{S}}-\dot{\mathrm{E}}(\mathbf{v}, \mathbf{u})) \mathrm{d} Q_{0} \\
& +\int_{\Omega_{0}} \delta \mathbf{v} \cdot\left(\rho_{0}\left(\mathbf{v}\left(t_{\mathrm{n}}^{+}\right)-\mathbf{v}\left(t_{\mathrm{n}}^{-}\right)\right)\right) \mathrm{d} \Omega_{0} \\
& +\int_{\Omega_{0}} \delta \mathrm{S}:\left([s]\left(\mathrm{S}\left(t_{\mathrm{n}}^{+}\right)-\mathrm{S}\left(t_{\mathrm{n}}^{-}\right)\right)\right) \mathrm{d} \Omega_{0} \\
& -\int_{\mathrm{P}_{0}^{\mathrm{t}}} \delta \mathbf{v} \cdot \overline{\mathbf{t}}_{0} \mathrm{~d} P_{0}=0 \quad \forall \delta \mathbf{v}, \delta \mathbf{S}
\end{aligned}
$$

where (23a) and (23b) are the integral forms of (7), weighted with velocity. The constitutive law given by (9), but without the coupling term, is solved in a weak sense on the element level leading to a mixed hybrid method. The jump terms in (23d) and (23e) allow for the consistent transfer of kinetic energy and internal mechanical energy between the time slabs $t_{\mathrm{n}}^{-}$and $t_{\mathrm{n}}^{+}$. The boundary tractions are considered in (23f). The displacement state $\mathbf{u}$ can be computed by the integration of the structural velocity $\mathbf{v}$. 


\subsection{Piezoelectric material}

The integral form of (7)-(11) including the piezoelectric coupling terms is given as

$$
\begin{aligned}
& \int_{\mathrm{Q}_{0, \mathrm{n}}} \delta \mathbf{v} \cdot \rho_{0} \dot{\mathbf{v}} \mathrm{d} Q_{0}+\int_{\mathrm{Q}_{0, \mathrm{n}}} \dot{\mathrm{E}}(\delta \mathbf{v}, \mathbf{u}): \operatorname{Sd} Q_{0} \\
& -\int_{\mathrm{Q}_{0, \mathrm{n}}} \delta \mathbf{v} \cdot \mathbf{f}_{0} \mathrm{~d} Q_{0} \\
& -\int_{\mathrm{Q}_{0, \mathrm{n}}} \dot{\tilde{\mathrm{E}}}_{0}(\delta \psi) \cdot \tilde{\mathrm{D}}_{0} \mathrm{~d} Q_{0} \\
& +\int_{\mathrm{Q}_{0, \mathrm{n}}} \delta \mathrm{S}:\left(\left[s^{\tilde{\mathrm{D}}}\right] \dot{\mathrm{S}}+[g]^{\top} \dot{\tilde{\mathrm{D}}}_{0}-\dot{\mathrm{E}}(\mathbf{v}, \mathbf{u})\right) \mathrm{d} Q_{0} \\
& +\int_{\mathrm{Q}_{0, \mathrm{n}}} \delta \tilde{\mathrm{D}}_{0} \cdot\left(-[g]^{\top} \dot{\mathrm{S}}+\left[\varepsilon^{\mathrm{S}}\right]^{-1} \dot{\tilde{\mathrm{D}}}_{0}-\dot{\tilde{\mathrm{E}}}_{0}(\psi)\right) \mathrm{d} Q_{0} \\
& +\int_{\Omega_{0}} \delta \mathbf{v} \cdot\left(\rho_{0}\left(\mathbf{v}\left(t_{\mathrm{n}}^{+}\right)-\mathbf{v}\left(t_{\mathrm{n}}^{-}\right)\right)\right) \mathrm{d} \Omega_{0} \\
& +\int_{\Omega_{0}} \delta \mathrm{S}:\left(\left[s^{\tilde{\mathrm{D}}}\right]\left(\mathrm{S}\left(t_{\mathrm{n}}^{+}\right)-\mathrm{S}\left(t_{\mathrm{n}}^{-}\right)\right)\right) \mathrm{d} \Omega_{0} \\
& +\int_{\Omega_{0}} \delta \mathrm{S}:\left([g]^{\top}\left(\tilde{\mathrm{D}}_{0}\left(t_{\mathrm{n}}^{+}\right)-\tilde{\mathrm{D}}_{0}\left(t_{\mathrm{n}}^{-}\right)\right)\right) \mathrm{d} \Omega_{0} \\
& +\int_{\Omega_{0}} \delta \tilde{\mathrm{D}}_{0} \cdot\left(-[g]\left(\mathrm{S}\left(t_{\mathrm{n}}^{+}\right)-\mathrm{S}\left(t_{\mathrm{n}}^{-}\right)\right)\right) \mathrm{d} \Omega_{0} \\
& +\int_{\Omega_{0}} \delta \tilde{\mathrm{D}}_{0} \cdot\left(\left[\varepsilon^{\mathrm{S}}\right]^{-1}\left(\tilde{\mathrm{D}}_{0}\left(t_{\mathrm{n}}^{+}\right)-\tilde{\mathrm{D}}_{0}\left(t_{\mathrm{n}}^{-}\right)\right)\right) \mathrm{d} \Omega_{0} \\
& -\int_{\mathrm{P}_{0}^{\mathrm{t}}} \delta \mathbf{v} \cdot \overline{\mathbf{t}_{0}} \mathrm{~d} P_{0}-\int_{P_{0}^{\psi}} \delta q \bar{\psi} d P_{0}=0 .
\end{aligned}
$$

As seen in the case of the purely elastic problem, the constitutive equations (9) and (14) are solved in a weak sense in (24c) and (24d) on element level leading to a mixed hybrid form with mechanical stress and electric displacement as element level unknowns. They are spatially discontinuous at element edges and can be condensed on the element level. Potential rate, $\psi$ is the additional global unknown field analogous to the velocity field.

\subsection{Circuit}

The integral form of the electrodes that cover the piezoelectric patches, and the harvester circuit attached to the electrodes are given as 


$$
\begin{array}{ll}
-\int_{\mathrm{P}_{0}^{\mathrm{E}}} \delta q \psi \mathrm{d} P_{0}-\int_{\mathrm{P}_{0}^{\mathrm{E}}} \delta \psi q \mathrm{~d} P_{0}+\int_{\mathrm{P}_{0}^{\mathrm{E}}} \delta q \dot{\Phi}_{\mathrm{p}} \mathrm{d} P_{0} & \forall \delta \psi, \delta q \\
-\int_{\mathrm{I}} \delta \Phi_{\mathrm{p}}\left(\frac{\Phi}{R}-\int_{\mathrm{P}_{0}^{\mathrm{E}}} \dot{q} \mathrm{~d} P\right) \mathrm{d} t=0 \quad \forall \delta \Phi_{\mathrm{p}} &
\end{array}
$$

where (25a) takes into account the charges localized on the continuous electrodes covering the patch, and the relation between the boundary charges and the single potential output of a piezoelectric patch is given in $(25 \mathrm{~b})$. These terms together naturally enforce the equipotential condition of the electrodes explained in Sect. 3.2 and (21).

\section{Discretization with space-time finite elements}

This subsection details the procedure involved in discretization of the domains constituting the problem setup using different types of finite elements. As a first step, an illustration of the different domains constituting the coupled system and the corresponding unknown fields is shown in Fig.3.

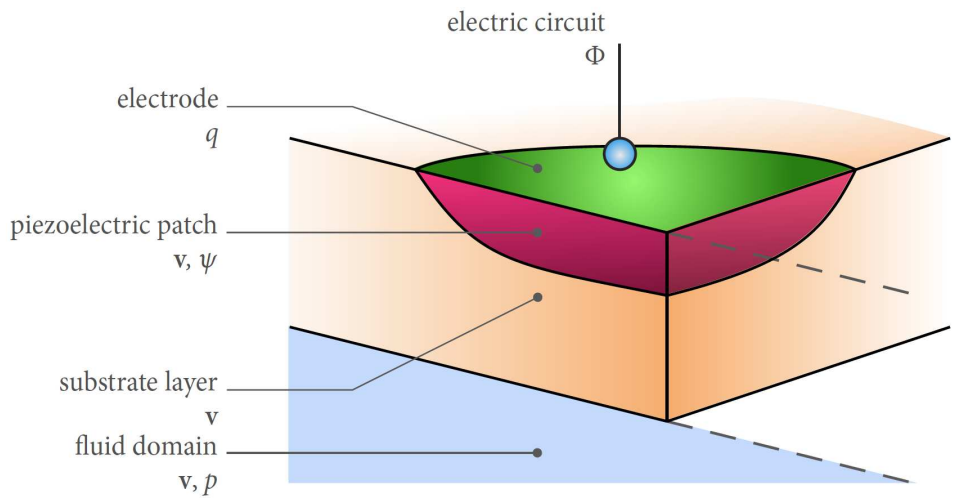

Fig. 3 An illustration of the coupled multi-physics domains with the associated unknown fields.

\subsection{Elements and space-time interpolation}

An eight node hexahedral element as depicted in Fig. 4 is chosen to discretize the fluid, structural and the piezoelectric weak forms spatially. Several works $[25,20,24,21]$ detail the application of the space-time finite element method for 
fluid-structure interaction. Since the electric charges are collected only on the surface of the electrodes, a four node quadrilateral element as shown in Fig. 5 is chosen to discretize the electrodes covering the piezoelectric patch. The quadrilateral element contains the electric charge $(q)$ and the potential rate $(\psi)$ as its degrees if freedom (dof). The electrode cover over the piezoelectric patch is assumed to be continuous. Thus each piezoelectric patch gives rise to a single potential output. This equipotential condition is enforced naturally in the formulation by coupling the nodal electrical potential rate d.o.f.s of the quadrilateral element to the virtual node containing the electrical potential degree of freedom $(\Phi)$. The potential output of the harvester is also the potential across the resistive element constituting the harvester circuit. This is explained in detail later in this section. The hexahedral ele-

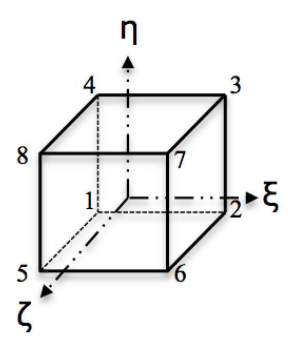

Fig. 4 An eight-node hexahedral element and its node numbering sequence

ment allows for a straightforward coupling of different domains (fluid, structure, and the piezoelectric material) without resorting to simplification of the problem under consideration. This approach also enables a strongly-coupled representation of the multi-physics problem. Locking phenomenon, which is encountered in modeling of thin structures using three dimensional elements is mitigated by adopting a mixed formulation. The thickness of the electrode is sufficiently smaller than the thickness of the piezoelectric patch, that the electrode layer is discretized using a four-node quadrilateral element.

The various assumed field variables within a generic finite element are discretized as

$$
\begin{aligned}
\mathbf{v} & =\mathrm{N}_{\mathbf{v}}^{\alpha} \mathbf{v}_{\mathrm{m}} \\
\psi & =\mathrm{N}_{\psi}^{\alpha} \psi_{\mathrm{e}} \\
\mathrm{S} & =\mathrm{N}_{\mathrm{S}}^{\alpha} \mathrm{S}_{\mathrm{m}} \\
\tilde{\mathrm{D}} & =\mathrm{N}_{\tilde{\mathrm{D}}}^{\alpha} \tilde{\mathrm{D}}_{\mathrm{e}} \\
q & =\mathrm{N}_{\mathrm{q}}^{\alpha} \mathbf{q}_{\mathrm{e}} \\
\Phi & =\mathrm{N}_{\Phi}^{\alpha} \Phi_{\mathrm{e}}
\end{aligned}
$$


where $\mathrm{N}_{\mathbf{v}}^{\alpha}$ in (26a) is the velocity interpolation matrix, and $\mathbf{v}_{\mathrm{m}}$ is the vector of nodal velocity d.o.f.s. $\mathrm{N}_{\psi}^{\alpha}$ in (26b) is the electric potential rate interpolation matrix, and $\psi_{\mathrm{e}}$ is vector of nodal electric potential rate dofs. $N_{\mathrm{S}}^{\alpha}$ in (26c) is the mechanical stress shape function matrix, and $S_{m}$ is the vector of stress co-efficients. $N_{\tilde{D}}^{\alpha}$ in $(26 d)$ is the electric displacement shape function matrix, and $\tilde{D}_{\mathrm{e}}$ is the vector of electric displacement coefficients. $N_{q}^{\alpha}$ in (26e) is the charge interpolation matrix, and $\mathbf{q}_{\mathrm{e}}$ is the vector of nodal charge d.o.f.s. $N_{\Phi}^{\alpha}$ in (26f) is the electric potential interpolation matrix, and $\Phi_{\mathrm{e}}$ is the vector of nodal electric potential d.o.f.s. The subscripts "e" and "m" refer to mechanical and electrical quantities respectively. The superscript " $\alpha$ " refers to the fact that the fields are interpolated in space and time. The velocity and electric potential rate satisfy the continuity requirements. The assumed mechanical stress and dielectric displacement are not expressed in terms of nodal values, but through unique shape functions and can be independent of the ones in other elements.

It is pertinent at this point to explain the nature of space-time interpolation. For the sake of clarity and brevity, the four-node space-time quadrilateral element shown in Fig. 5 is taken as an example to briefly explain the derivation of space-time interpolation functions. For a more extensive study, readers are referred to [24, 22]. The extension of the concept to an eight-node hexahedral element is fairly straightforward. Typical shape functions used in spatial finite elements have an additional temporal component in space-time finite elements. For the four-node quadrilateral element, the spatial and temporal components of the typical space-time shape functions are given by

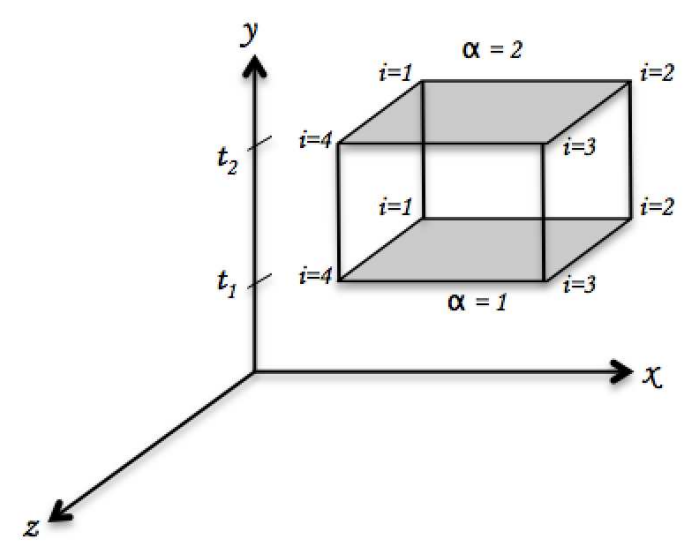

Fig. 5 A four-node quadrilateral space-time element

$$
N_{\mathrm{i}}^{\alpha}=N_{\mathrm{i}} T^{\alpha}=N_{\mathrm{i}}(\xi, \eta) T^{\alpha}(\theta),
$$


where " $i=1,2, \ldots n_{\text {nodes }}$ " refers to the number of nodes and " $\alpha=1,2, .$. " refers to the temporal division of the time slab. $\xi, \eta \in[-1,+1]$ are the natural spatial coordinates, and $\theta \in[-1,+1]$ is the natural temporal co-ordinate.

Temporal shape function can be explicitly defined as opposed to the spatial shape functions which usually depends on the spatial dimensions. The temporal shape function is expressed as

$$
T^{1}(\theta)=\frac{1}{2}(1-\theta), \quad T^{2}(\theta)=\frac{1}{2}(1+\theta) .
$$

Time derivative of any unknown field can be readily obtained by taking the derivative of (28) and is expressed as

$$
T_{, \theta}^{1}=-\frac{1}{2}, \quad T_{, \theta}^{2}=+\frac{1}{2} .
$$

The spatial co-ordinates $x$ and $y$ can be interpolated in space and time as given by the following equations assuming linear interpolation in time

$$
\begin{aligned}
x & =\sum_{\alpha=1}^{\alpha=2 \mathrm{i}=\mathrm{n}} \sum_{\mathrm{i}=1}^{\alpha} N_{\mathrm{i}}^{\alpha} x_{\mathrm{i}}^{\alpha}=\sum_{\alpha=1}^{\alpha=2 \mathrm{i}=\mathrm{n}} \sum_{\mathrm{i}=1}^{\mathrm{i}=\mathrm{n}} N_{\mathrm{i}} T^{\alpha} x_{\mathrm{i}}^{\alpha} \\
& =\sum_{\mathrm{i}=1}^{\mathrm{i}=\mathrm{n}} N_{\mathrm{i}}\left(T^{1} x_{\mathrm{i}}^{1}+T^{2} x_{\mathrm{i}}^{2}\right)=\sum_{\mathrm{i}=1}^{\mathrm{i}=\mathrm{n}} N_{\mathrm{i}} x_{\mathrm{i}}(\theta) \\
& =\sum_{\mathrm{i}=1}^{\mathrm{n}} N_{\mathrm{i}} x_{\mathrm{i}}
\end{aligned}
$$

and

$$
\begin{aligned}
& y=\sum_{\alpha=1}^{\alpha=2} \sum_{\mathrm{i}=1}^{\mathrm{i}=\mathrm{n}} N_{\mathrm{i}}^{\alpha} y_{\mathrm{i}}^{\alpha}=\sum_{\alpha=1}^{\alpha=2 \mathrm{i}=\mathrm{n}} \sum_{\mathrm{i}=1} N_{\mathrm{i}} T^{\alpha} y_{\mathrm{i}}^{\alpha} \\
& =\sum_{\mathrm{i}=1}^{\mathrm{i}=\mathrm{n}} N_{\mathrm{i}}\left(T^{1} y_{\mathrm{i}}^{1}+T^{2} y_{\mathrm{i}}^{2}\right)=\sum_{\mathrm{i}=1}^{\mathrm{i}=\mathrm{n}} N_{\mathrm{i}} y_{\mathrm{i}}(\theta) \\
& =\sum_{\mathrm{i}=1}^{\mathrm{i}=\mathrm{n}} N_{\mathrm{i}} y_{\mathrm{i}} \text {. }
\end{aligned}
$$

Similarly, we can interpolate the temporal co-ordinate $t$, where $t_{\mathrm{i}}^{\alpha}=t^{\alpha}$ for " $i=$ $1,2,3, \ldots n$ " as

$$
\begin{aligned}
t & =\sum_{\alpha=1}^{\alpha=2} \sum_{\mathrm{i}=1}^{\mathrm{i}=\mathrm{n}} N_{\mathrm{i}}^{\alpha} T_{\mathrm{i}}^{\alpha}=\sum_{\alpha=1}^{\alpha=2} \sum_{\mathrm{i}=1}^{\mathrm{i}=\mathrm{n}} N_{\mathrm{i}} T^{\alpha} t^{\alpha} \\
& =\left(\sum_{\mathrm{i}=1}^{\mathrm{i}=\mathrm{n}} N_{\mathrm{i}}\right) \sum_{\alpha=1}^{\alpha=2} T^{\alpha} t^{\alpha}=T^{1} t^{1}+T^{2} t^{2}
\end{aligned}
$$


The derivative of the shape functions with respect to the global axis is given by the following equations

$$
\begin{aligned}
{\left[\begin{array}{l}
N_{\mathrm{a}, \mathrm{x}}^{\alpha} \\
N_{\mathrm{a}, \mathrm{y}}^{\alpha} \\
N_{\mathrm{a}, \mathrm{t}}^{\alpha}
\end{array}\right]=} & {\left[\begin{array}{lll}
\xi_{, \mathrm{x}} & \eta_{, \mathrm{x}} & \theta_{, \mathrm{x}} \\
\xi_{, \mathrm{y}} & \eta_{, \mathrm{y}} & \theta_{, \mathrm{y}} \\
\xi_{, \mathrm{t}} & \eta_{, \mathrm{t}} & \theta_{, \mathrm{t}}
\end{array}\right]\left[\begin{array}{l}
N_{\mathrm{a}, \xi}^{\alpha} \\
N_{\mathrm{a}, \eta}^{\alpha} \\
N_{\mathrm{a}, \theta}^{\alpha}
\end{array}\right] } \\
= & {\left[\begin{array}{lll}
x_{, \xi} & y_{, \xi} & t_{, \xi} \\
x_{, \eta} & y_{, \eta} & t_{, \eta} \\
x_{, \theta} & y_{, \theta} & t_{, \theta}
\end{array}\right]^{-1}\left[\begin{array}{l}
N_{\mathrm{a}, \xi}^{\alpha} \\
N_{\mathrm{a}, \eta}^{\alpha} \\
N_{\mathrm{a}, \theta}^{\alpha}
\end{array}\right], }
\end{aligned}
$$

where in (33b) the derivative of global time axis with respect to the local spatial axes is zero $t_{, \xi}, t_{, \eta}=0$. Also, the structure is modeled in Lagrangian framework and hence the derivative of global axes with respect to the local time axes is zero $x_{, \theta}, y_{, \theta}=0$ and $t_{, \theta}=\frac{\Delta t}{2}$.

With this brief introduction in place, for the eight-node hexahedral element shown in Figure 4, the space-time interpolation function for the $i$ th node can expressed as

$$
N_{\mathrm{i}}^{\alpha}=N_{\mathrm{i}} T^{\alpha}=N_{\mathrm{i}}(\xi, \eta, \zeta) T^{\alpha}(\theta)
$$

where $N_{\mathrm{i}}, i=1, \ldots, n_{\text {nodes }}$, as seen earlier, is the spatial interpolation function and is given by

$$
N_{\mathrm{i}}=\frac{1}{8}\left(1+\xi_{\mathrm{i}} \xi\right)\left(1+\eta_{\mathrm{i}} \eta\right)\left(1+\zeta_{\mathrm{i}} \zeta\right)
$$

in which $\xi, \eta$ and $\zeta \in[-1,+1]$ are the natural spatial co-ordinates.

The assumed field variables potential rate and velocity can be interpolated as

$$
\begin{aligned}
\psi & =\left[T^{1}\left[N_{1}, \ldots, N_{8}\right] T^{2}\left[N_{1}, \ldots, N_{8}\right]\right]\left\{\psi_{1}, \ldots \psi_{16}\right\}^{\top} \\
& =\mathrm{N}_{\psi}^{\alpha} \psi_{\mathrm{e}} \\
\mathbf{v} & =\left[T^{1}\left[\left.N_{1}\right|_{3}, \ldots,\left.N_{8}\right|_{3}\right] T^{2}\left[N_{1} \mathrm{I}_{3}, \ldots, N_{8} \mathrm{I}_{3}\right]\right]\left\{v_{1}, \ldots v_{48}\right\}^{\top} \\
& =\mathrm{N}_{\mathbf{v}}^{\alpha} \mathbf{v}_{\mathrm{m}}
\end{aligned}
$$

where in (36a) and (36c), it can be seen that the are 16 potential rate dofs, and 48 velocity dofs.respectively. This is because the interpolation is performed in space and time. This is true in case of all the field variables. In (36c), $\mathrm{I}_{\mathrm{i}}$ is the $i$ th order identity matrix.

The mechanical stress and electric displacement are approximated using unique shape functions. For the mechanical stress, the stress shape function used in the Pian's hybrid element [26, 27, 28] is used. Pian's hybrid element contains 18 stress modes. For the electric displacement, the shape function employed in [13] is used. Sze's element contains 7 assumed electric displacement modes. In a space-time setting, the mechanical stress and electric displacement will have 36 and 14 assumed 
modes respectively. The number of assumed mechanical stress and electric displacement modes are chosen so to secure proper element rank.

The interpolation of the mechanical stress can be expressed as

$$
\begin{aligned}
\mathrm{S} & =\left[T^{1}\left[\mathrm{I}_{6} \mathrm{~T}_{\mathrm{m}} \mathrm{N}_{\mathrm{S}}\right] T^{2}\left[\mathrm{I}_{6} \mathrm{~T}_{\mathrm{m}} \mathrm{N}_{\mathrm{S}}\right]\right]\left\{S_{1}, \ldots, S_{36}\right\}^{\top} \\
& =\mathrm{N}_{\mathrm{S}}^{\alpha} \mathrm{S}_{\mathrm{m}}
\end{aligned}
$$

where $T_{m}$ is the transformation matrix evaluated at the element origin, I is the identity matrix, and $\mathrm{N}_{\mathrm{S}}$ is the stress shape function matrix and given as

$$
\left[\begin{array}{cccccccccccc}
0 & 0 & 0 & \eta & 0 & 0 & \zeta & 0 & 0 & \eta \zeta & 0 & 0 \\
\xi & 0 & 0 & 0 & 0 & 0 & 0 & \zeta & 0 & 0 & \zeta \xi & 0 \\
0 & \xi & 0 & 0 & \eta & 0 & 0 & 0 & 0 & 0 & 0 & \xi \eta \\
0 & 0 & 0 & 0 & 0 & 0 & 0 & 0 & \zeta & 0 & 0 & 0 \\
0 & 0 & \xi & 0 & 0 & 0 & 0 & 0 & 0 & 0 & 0 & 0 \\
0 & 0 & 0 & 0 & 0 & \eta & 0 & 0 & 0 & 0 & 0 & 0
\end{array}\right]
$$

The electrical displacement shape function as given in [13], in a space-time setting, can be expressed as

$$
\tilde{\mathrm{D}}=\left[T^{1}\left[\mathrm{I}_{3} \mathrm{~T}_{\mathrm{e}} \mathrm{N}_{\tilde{\mathrm{D}}}\right] T^{2}\left[\mathrm{I}_{3} \mathrm{~T}_{\mathrm{e}} \mathrm{N}_{\tilde{\mathrm{D}}}\right]\right]\left\{D_{1}, \ldots, D_{14}\right\}^{\top},
$$

where in (39), $T_{e}$ is the Jacobian matrix evaluated at the origin of the natural coordinates, and $\mathrm{N}_{\tilde{\mathrm{D}}}$ is the electric displacement shape function as given by

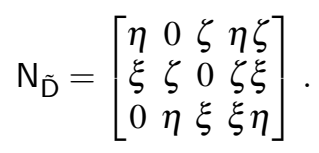

As mentioned earlier, the four-node quadrilateral space-time element shown in Figure 5 is employed as a boundary element to discretize the charges localized on the electrode surface. For this element, the space-time interpolation function for the $i$ th node is given in (27), where

$$
N_{\mathrm{i}}=\frac{1}{4}\left(1+\xi_{\mathrm{i}} \xi\right)\left(1+\eta_{\mathrm{i}} \eta\right) .
$$

The electric charge can be interpolated as,

$$
\begin{aligned}
q & =\left[T^{1}\left[N_{1}, \ldots, N_{4}\right] T^{2}\left[N_{1}, \ldots N_{4}\right]\right]\left\{q_{1}, \ldots, q_{8}\right\}^{\top} \\
& =\mathrm{N}_{\mathrm{q}}^{\alpha} \mathbf{q}_{\mathrm{e}} .
\end{aligned}
$$

The electric potential is discretized only in time domain to meet the equipotential condition. It is represented by a single virtual node in space, and thus has a unit shape function in space. The space-time interpolation of the electric potential is given expressed as 


$$
\begin{aligned}
\Phi & =\left[T^{1}\left[N_{1}\right] T^{2}\left[N_{1}\right]\right]\left\{\Phi_{1}, \Phi_{2}\right\}^{\top} \\
& =\mathrm{N}_{\Phi}^{\alpha} \Phi_{e}
\end{aligned}
$$

where $N_{1}$ in (43a) is 1 .

The expressions for mechanical strain rate $(\dot{\mathbf{E}})$ in $(8)$ and electric field rate $(\dot{\tilde{\mathbf{E}}})$ in (13) can be obtained by differentiating (36d) and (36b) respectively, and expressed as

$$
\begin{array}{r}
\dot{\mathrm{E}}=\mathrm{B}_{\mathbf{v}}^{\alpha} \mathbf{v}_{\mathrm{m}} \\
\dot{\tilde{\mathrm{E}}}=\mathrm{B}_{\psi}^{\alpha} \psi_{\mathrm{e}} .
\end{array}
$$

As mentioned earlier, time derivative of any unknown field can easily be obtained by taking the derivative of time interpolation function as given in (29) and multiplying the spatial interpolation function. As an example, time derivative of velocity can be expressed as follows:

$$
\begin{aligned}
\dot{\mathbf{v}} & =\left[T_{, \theta}^{1}\left[\mathrm{~N}_{\mathbf{v}}\right] T_{, \theta}^{2}\left[\mathrm{~N}_{\mathbf{v}}\right]\right]\left\{v_{1}, \ldots, v_{48}\right\}^{\top} \\
& =\dot{\mathrm{N}}_{\mathbf{v}}^{\alpha} \mathbf{v}_{\mathrm{m}} .
\end{aligned}
$$

Time derivative of other unknown fields can be obtained in the same way as shown above.

\subsection{Monolithic solution strategy}

At the element level, the space-time discretization of a specific time slab $Q_{n}$ applied to the weak form of the coupled system (22a) - (25b), leads to a system of coupled algebraic equations:

$$
\mathrm{K}_{\text {lin }}\left(\mathbf{v}_{\mathrm{i}}, \psi_{\mathrm{i}}, q_{\mathrm{i}}, \Phi_{\mathrm{i}}, \mathrm{S}, \tilde{\mathrm{D}}\right) \Delta \mathbf{x}=\mathbf{r}
$$

where $\mathrm{K}_{\text {lin }}$ is the element level coefficient matrix, $\Delta \mathbf{x}$ is the vector of unknowns, and $\mathbf{r}$ is the residual vector. The mechanical stress and the electric displacement are discontinuous across the elements, they can be statically condensed on the element level. The resulting element-level matrices are assembled in the usual way by nodal addition of elemental contributions and can be expressed as

$$
\mathrm{K}_{\text {lin }}^{*}\left(\mathbf{v}_{\mathrm{i}}, \psi_{\mathrm{i}}, q_{\mathrm{i}}, \Phi_{\mathrm{i}}\right) \Delta \mathbf{x}^{*}=\mathbf{r}^{*}
$$

where $\mathrm{K}_{\text {lin }}^{*}$ is the global coefficient matrix, $\Delta \mathbf{x}^{*}$ is the vector of global unknowns, and $\mathbf{r}^{*}$ is the global residual vector.

Equation (47) is a monolithic algebraic representation of the discretized coupled multi-physics problem, and the solution to the unknown fields as shown in Fig.6 is obtained using the Newton-Raphson iterative scheme. 


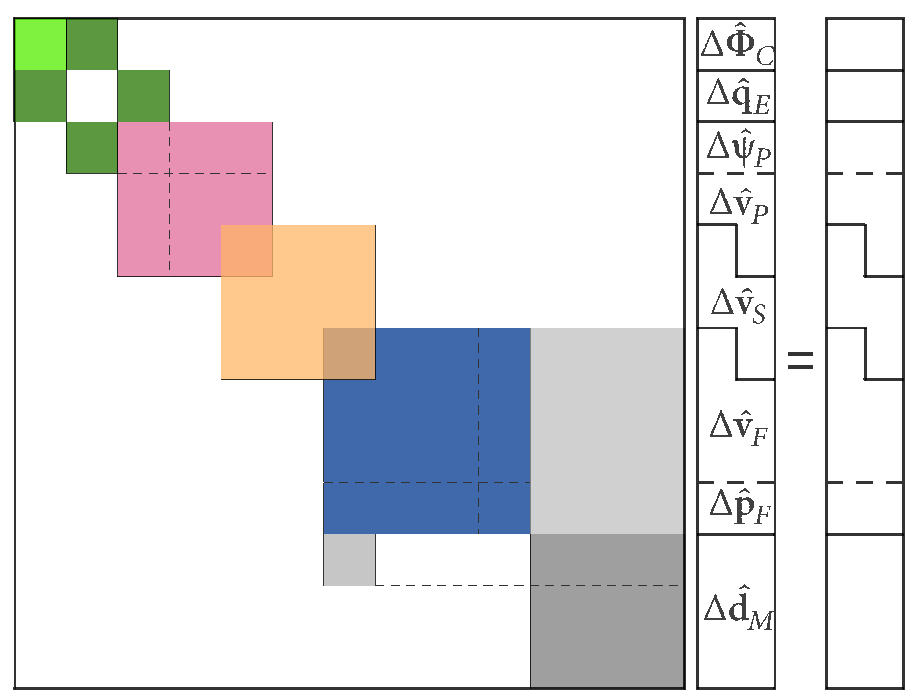

Fig. 6 Structure of the monolithic algebraic system including mesh-deformation d.o.f.s.

\section{Numerical Example}

This section is devoted to the application of theoretical concepts explained in the preceding sections to the problem of piezoelectric energy harvesting from base excitations.

\subsection{Problem setup}

The bimorph cantilever beam considered in this numerical example is also discussed in [8], and the basic setup is shown in Fig. 7 The substructure is sandwiched between two identical piezo patches which are fully covered with conductive electrodes. The piezo patches are polarized in the same direction thus constituting a parallel connection between the electrodes. A harvesting circuit constituting a resistor $R$ is also attached to the electrodes. The base excitation causes longitudinal strains ( $x$ direction) in the beam which are coupled to the electric field in transverse direction ( $y$ direction) leading to a -31 coupling of the piezoelectric elements. The geometric and material properties of the piezoceramic and substrate layers are given in Table 1. In Table 1 , the permittivity $\varepsilon_{33}^{\mathrm{E}}\left(\varepsilon_{0}=8.854 \mathrm{pF} \mathrm{m}^{-1}\right)$ is the measure at constant strain and piezoelectric voltage coefficient $d_{31}$ is the measure used in strain-charge form of the piezoelectric constitutive equation. However, the constitutive relation given in equations (9) and (14) is expressed in terms of $\dot{E}$ and $\dot{\tilde{E}}$ as a function of S and $\tilde{D}$. 


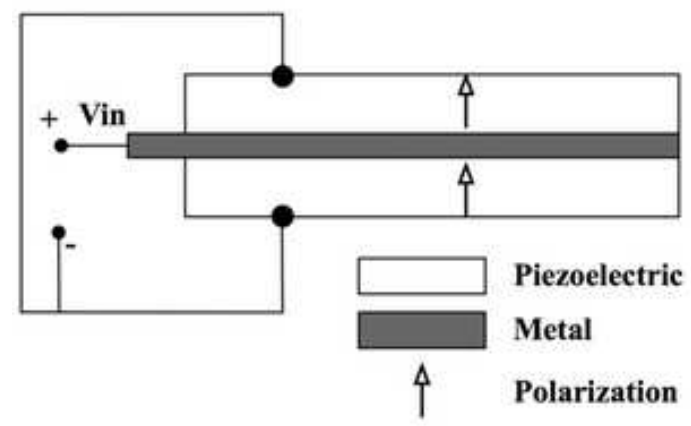

Fig. 7 Parallel configuration of piezoelectric bimorph [29]

Table 1 Geometric and material properties of the energy harvester

\begin{tabular}{|c|c|c|}
\hline Quantity & Dimension & Value \\
\hline Length of the beam & $L(\mathrm{~mm})$ & 50.8 \\
\hline Width of the beam & $b(\mathrm{~mm})$ & 31.8 \\
\hline $\begin{array}{l}\text { Thickness of the piezo. patch } \\
\text { (PZT-5A) }\end{array}$ & $h_{\mathrm{p}}(m m)$ & 0.26 (each) \\
\hline Thickness of the substructure & $h_{\mathrm{s}}(\mathrm{mm})$ & 0.14 \\
\hline $\begin{array}{l}\text { Young's modulus of the } \\
\text { substructure (brass) }\end{array}$ & $Y_{\mathrm{s}}(G P a)$ & 105 \\
\hline $\begin{array}{l}\text { Young's modulus of } \\
\text { PZT-5A }\end{array}$ & $Y_{\mathrm{p}}(G P a)$ & 66 \\
\hline $\begin{array}{l}\text { Mass density of the } \\
\text { substructure (brass) }\end{array}$ & $\rho_{\mathrm{s}}\left(K g m^{-3}\right)$ & 9000 \\
\hline $\begin{array}{l}\text { Mass density of } \\
\text { PZT-5A }\end{array}$ & $\rho_{\mathrm{p}}\left(K g m^{-3}\right)$ & 7800 \\
\hline $\begin{array}{l}\text { Piezoelectric displacement coef- } \\
\text { ficient }\end{array}$ & $d_{31}\left(p m V^{-1}\right)$ & -190 \\
\hline Permittivity & $\varepsilon_{33}^{\mathrm{E}}\left(F m^{-1}\right)$ & $1500 \varepsilon_{0}$ \\
\hline
\end{tabular}

The following relations are used to transform the values in the table to fit the formulation given in (9) and (14).

$$
\begin{aligned}
{\left[\varepsilon^{\mathrm{S}}\right] } & =\left[\varepsilon^{\mathrm{E}}\right]+[d]\left[c^{\tilde{\mathrm{E}}}\right][d]^{\top} \\
{\left[\varepsilon^{\mathrm{S}}\right]^{-1} } & =\left(\left[\varepsilon^{\mathrm{E}}\right]+[d]\left[c^{\tilde{\mathrm{E}}}\right][d]^{\top}\right)^{-1},
\end{aligned}
$$

where $\left[c^{\tilde{\mathrm{E}}}\right]$ is the Young's modulus $Y_{p}$ of the piezoelectric material measured at constant electric field as given in table. 
The compliance matrix at constant dielectric displacement is obtained from the table values as follows

$$
\left[s^{\tilde{\mathrm{D}}}\right]=\left[c^{\tilde{\mathrm{E}}}\right]^{-1}-\left([d]^{\top}\left[\varepsilon^{\mathrm{S}}\right]^{-1}[d]\right)
$$

The piezoelectric voltage coefficient derived from displacement coefficient is given by

$$
[g]=\left[\varepsilon^{S}\right]^{-1}[d]
$$

The substructure is discretized using a three dimensional mixed-hybrid spacetime structural element, and the piezoelectric material is discretized using a three dimensional mixed-hybrid space-time piezoelectric element. The electrodes on the top and the bottom surfaces of the bimorph are discretized using a spatially twodimensional space-time face element, and the harvester circuit is attached to the electrode elements by coupling them with a common virtual node. The length dimension of the bimorph is discretized using 15 elements, the width dimension with 2 elements. The substructure is discretized using 2 elements in the height dimension and the piezoelectric material with 3 elements. Since the substructure is sandwiched between two piezoelectric patches, the total number of elements in the height dimension of the bimorph is 8 .

The parallel connection of the conductive electrodes is facilitated by having the same polarization direction for the top and bottom piezo elements. Physically this means that both the top and bottom surfaces of the bimorph constitute one terminal and the electrode layers present in the upper piezo-substructure interface and lower piezo-substructure interface constitute the other terminal. The interface terminal is grounded by setting the nodes of the piezoelectric element to zero. The potential on the top surface is equal to the potential of the bottom surface, and this potential drives the harvesting circuit represented by a resistor element.

In harvesting energy from base excitations, many studies focus on the excitation of the harvester at it's fundamental resonance frequency to investigate power output characteristics of the harvester. The first fundamental short circuit $(R=0)$ frequency of the piezoelectric bimorph considered in this study is $118.5 \mathrm{~Hz}$. The bimorph is excited at this frequency to observe the power output and vibration characteristics.

Fig. 8 presents the evolution of electrical power and electrical potential with time at two different resistances. Since it is impractical to have exactly zero resistance under experimental conditions, a resistance of $1 K \Omega$ is chosen to represent short circuit condition. The harvester reaches a steady state voltage of $4 \mathrm{~V}$ at short circuit condition compared to its steady stage voltage output of $6 \mathrm{~V}$ at $R=10 \mathrm{~K} \Omega$. This behavior is expected as potential builds up in the electrodes when there is infinite resistance present between the terminals, and the electrical potential drops to zero when there is zero resistance present between the terminals. Moreover, the steady state power output of $4 \mathrm{~mW}$ is reasonable for the given base excitation. Fig. 9 presents the evolution of relative tip displacement and base excitation with time. The results suggests that when a finite resistance is present between the terminals, the displacement am- 

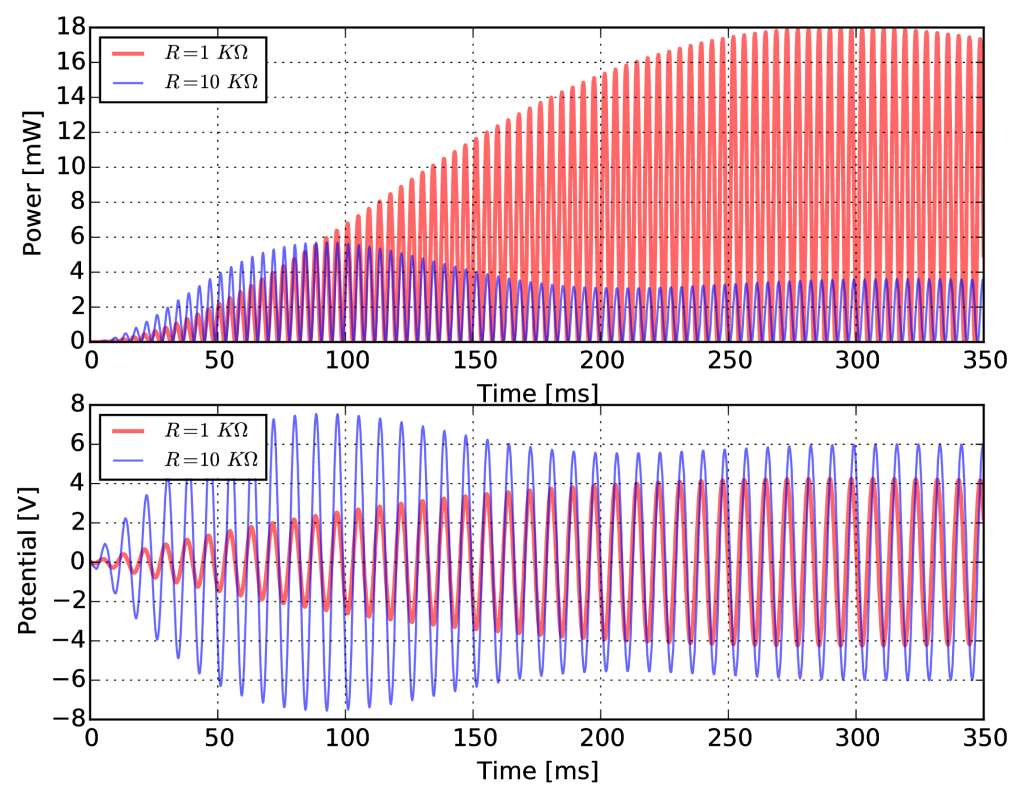

Fig. 8 Solution for electrical power (top) and electrical potential (bottom) with time.

plitudes are suppressed. This is due to the fact that the attached circuit has an impact on the vibration characteristics of the harvester, and this impact can be captured effectively in a strongly-coupled modeling approach as presented in this study.

\section{Acknowledgements}

The authors gratefully acknowledge the support of the FNR AFR PhD grant 3996097 and the FP7 Marie Curie - Career Integration Grant 322151 by the European Commission.

\section{References}

1. Steven R Anton and Henry A Sodano. A review of power harvesting using piezoelectric materials (20032006). Smart materials and structures, 16:1-21, 2007.

2. H A Sodano, G Park, and D J Inman. Estimation of electric charge output for piezoelectric energy harvesting. Strain, 40:49-58, 2004.

3. Jessy Baker, Shad Roundy, and Paul Wright. Alternative geometries for increasing power density in vibration energy scavenging for wireless sensor networks. Proceedings of the 3rd 


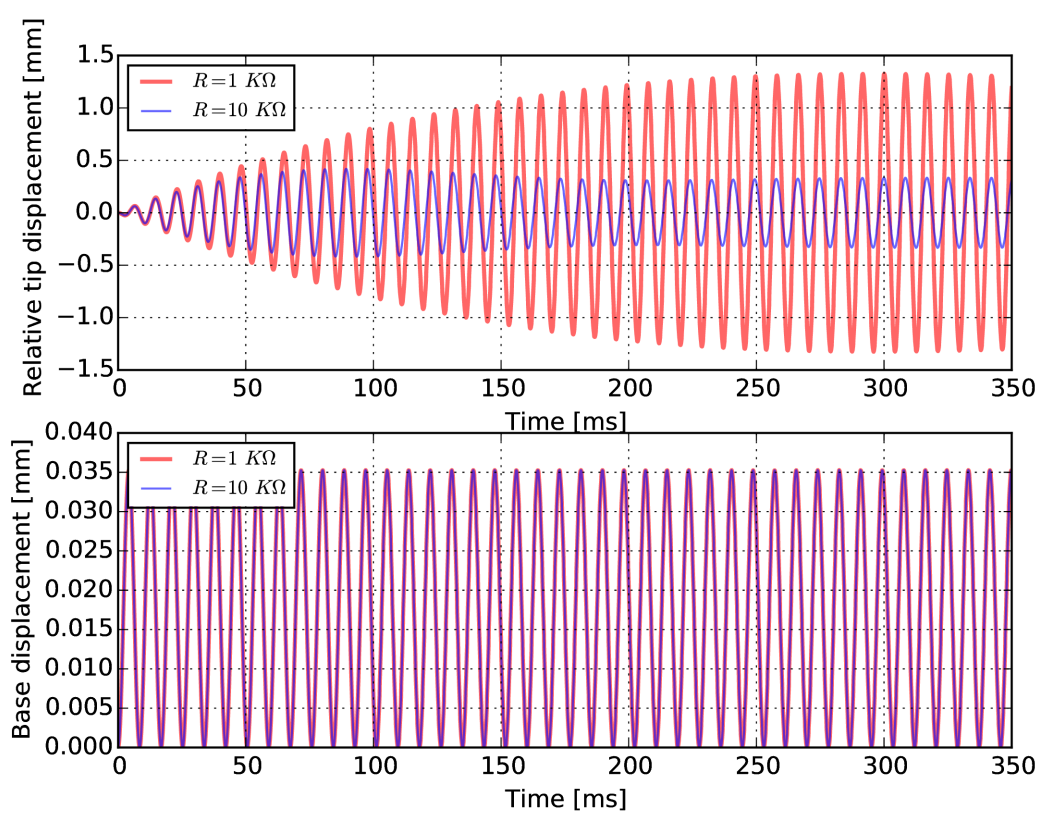

Fig. 9 Solution for tip velocity (top) and relative end displacement (bottom) with time.

international enery conversion conference, pages 1-12, 2005.

4. Henry A Sodano, Justin Lloyd, and Daniel J Inman. An experimental comparison between several active composite actuators for power generation. Smart material structures, 15:12111216, 2006.

5. Y Yang and L Tang. Equivalent circuit modeling of piezoelectric energy harvesters. Intelligent material systems and structures, 20:2223-2235, 2009.

6. N G Elvin and Alex A Elvin. A general equivalent circuit model for piezoelectric generators. Intelligent material systems and structures, 20:3-9, 2009.

7. D J Erturk, Aand Inman. On mechanical modeling of cantilevered piezoelectric vibration energy harvesters. Intelligent material systems and structures, 19:1311-1325, 2008.

8. A Erturk and D J Inman. An experimentally validated bimorph cantilever model for piezoelectric energy harvesting from base excitations. Smart materials and structures, 18:1-18, 2009.

9. O Thomas, J F Deü, and J Ducarne. Vibrations of an elastic structure with shunted piezoelectric patches: efficient finite element formulation and electromechanical coupling coefficients. Numerical methods in engineering, 80:235-268, 2009.

10. H Allik and Thomas J R Hughes. Finite element method for piezoelectric vibration. Numerical methods in engineering, 2:151-157, 1970.

11. A Benjeddou. Advances in piezoelectric finite element modeling of adaptive structural elements: a survey. Computers and structures, 76:347-363, 2000.

12. J S Yang. Equations for thick elastic plates with partially electroded piezoelectric actuators and higher order electric fields. Smart materials and structures, 8, 1999.

13. K Y Sze and Y S Pan. Hybrid finite elementmodels for piezoelectric materials. Sound and vibration, 226:519-547, 1999.

14. C C Wu, K Y Sze, and Y Q Huang. Numerical solutions on fracture of piezoelectric materials by hybrid element. Solids and structures, 38:4315-4329, 2001. 
15. S Klinkel and W Wagner. A geometrically non-linear piezoelectric solid shell element based on a mixed multi-field variational formulation. Numerical methods in engineering, 65:349$382,2006$.

16. Sven Klinkel, Friedrich Gruttmann, and Werner Wagner. A robust non-linear solid shell element based on a mixed variational formulation. Computer methods in applied mechanics and engineering, 195:179-201, 2006.

17. J J Allen and A J Smits. Energy harvesting eel. Fluid and structures, 15:629-640, 2001.

18. G W Taylor, J R Burns, S a Kammann, W B Powers, and T R Welsh. The energy harvesting eel: a small subsurface ocean/river power generator. IEEE journal of oceanic engineering, 26:539-547, 2001.

19. Huseyin Dogus Akaydin, Niell Elvin, and Yiannis Andreopoulos. Energy harvesting from highly unsteady fluid flows using piezoelectric materials. Intelligent material systems and structures, 21:1263-1278, 2010.

20. B Hübner and D Dinkler. A simultaneous solution procedure for strong interactions of generalized newtonian fluids and viscoelastic solids at large strains. Numerical methods in engineering, 64:920-939, 2005.

21. A Zilian and A Legay. The enriched space-time finite element method (EST) for simultaneous solution of fluid-structure interaction. Numerical methods in engineering, 75:305-334, 2008.

22. Thomas J R Hughes and Gregory M Hulbert. Space-time finite elmement methods for elastodynamics: formulations and error estimates. Computer methods in applied mechanics and engineering, 66:339-363, 1988.

23. Gregory M Hulbert and Thomas J R Hughes. Space-time finite element methods for secondorder hyperbolic equations. Computer methods in applied mechanics and engineering, 84:327-348, 1990.

24. Tayfun E Tezduyar, Sunil Sathe, Ryan Keedy, and Keith Stein. Spacetime finite element techniques for computation of fluid-structure interactions. Computer methods in applied mechanics and engineering, 195:2002-2027, 2006.

25. E Walhorn, A Kölke, B Hübner, and D Dinkler. Fluid-structure coupling within a monolithic model involving free surface flows. Computers and structures, 83:2100-2111, 2005.

26. Theodore H H Pian and Da-Peng Chen. Alternative ways for formulation of hybrid stress elements. Numerical methods in engineering, 18:1679-1684, 1982.

27. Theodore H H Pian and K Sumihara. Roelements approach for assumed stress finite elements. Numerical methods in engineering, 20:1685-1695, 1984.

28. Theodore H H Pian. State-of-the-art development of hybrid/mixed finite element method. Finite elements in analysis and design, 21:5-20, 1995.

29. Hyeong Jae Lee, Shujun Zhang, Yoseph Bar-Cohen, and Stewart Sherrit. High temperature, high power piezoelectric composite transducers. Sensors, 14:14526-14552, 2014. 